\title{
Assessment of Prothrombin Time, Activated Partial Thromboplastic Time Platelets Count and Serum Alpha Amylase in Acute Pancreatitis Sudanese Patients
}

\author{
Amna Siddig Hamdan Obied ${ }^{1}$, Dr. Amira Ahmed Khalid Humeida ${ }^{2}$ \\ ${ }^{I}$ Department of Hematology, Faculty of Medical Laboratory Sciences, Al-Neelain University, Khartoum, \\ Sudan. \\ ${ }^{2}$ Assistant Professor , Department of Pathology, Faculty of Medicine, Al-Neelain University, Khartoum, Sudan.
}

\begin{abstract}
:
Background: Acute pancreatitis is a sudden inflammation that lasts for a short time. It may range from mild discomfort to a severe, life-threatening illness.Acute pancreatitis (AP) is a potentially lethal disorder with no specific medical treatment. AP is characterized by a spectrum of symptoms, ranging from a local inflammatory process to more severe form (acute necrotizing pancreatitis).

In addition, pancreatitis may be further classified into acute interstitial and acute hemorrhagic disease. In the first type, the gland architecture is preserved but is edematous. Inflammatory cells and interstitial oedema are prominent within the parenchyma. Haemorrhagic disease is characterized by marked necrosis, haemorrhage of the tissue, and fat necrosis There is marked pancreatic necrosis along with vascular inflammation and thrombosis. Severe acute pancreatitis may cause dehydration and low blood pressure. The heart, lungs, or kidneys may fail and if bleeding occurs in the pancreas, shock and even death may follow

Objective: The purpose of this study was to assess of Prothrombin Time, Activated Partial Thromboplastic Time, Platelets count and serum amylase in acute pancreatitis Sudanese patients.

Materials and Methods: This study a case control study, that included Sixty samples to assess Prothrombin Time(PT), Activated Partial Thromboplastic Time(APTT), Platelets countand serum amylase in acute pancreatitis Sudanese patients. 30 samples were collected from patients (case group) and 30 samples were collected from healthy persons which represented the control group.This study was approved by ethical committee of ministry of health, and informed consent was obtained from each participant before sample collection.Data was be collected by a structured questionnaire system from patients inIbnSenahospital. The PT ,APTT was done by semi-automated coagulometer (COATRON-MI) and platelate by using Sysmex Kx-21N.the data analyzed using SPSS21.

Results: In this study the mean $\pm S D$ of age PT, PTT and Platelets in case group were59.33 ( \pm 7.36$), 31.06$ ( \pm $6.29)$ seconds, $57.33( \pm 9.20)$ seconds and $306.53( \pm 60.44) \times 10^{9} / \mu L$ respectively, While the mean $\pm S D$ of PT, PTT and Platelets in control group were $13.33( \pm 1.15)$ seconds, $34.94( \pm 2.59)$ seconds and $287.43( \pm$ 47.59) $\times 10^{9} / \mu$ Lrespectively. the difference was statistically significant $(P=0.00)$
\end{abstract}

Conclusion: This study concluded that PT and APTT were prolonged in patients with acute pancreatitis compared to the normal subjects and Platelets count was within normal range in all patients.

Keywords: Sudanese, Acute pancreatitis, PT, APTT, Platelets.

\section{Introduction}

Acute pancreatitis is a sudden inflammation that lasts for a short time. It may range from mild discomfort to a severe, life-threatening illness. Most people with acute pancreatitis recover completely after getting the right treatment. In severe cases, acute pancreatitis can result in bleeding into the gland, serious tissue damage, infection, and cyst formation. Severe pancreatitis can also harm other vital organs such as the heart, lungs, and kidneys. ${ }^{[1]}$

\section{Symptoms of acute pancreatitis:}

The most common symptoms and signs include:

- severe epigastric pain (upper abdominal pain) radiating to the back in $50 \%$ of cases

- nausea

- vomiting

- loss of appetite

- fever

- chills (shivering)

- hemodynamic instability, including shock 
- tachycardia (rapid heartbeat)

- respiratory distress

- peritonitis

- $\quad$ hiccup[2]

Although these are common symptoms, they are not always present. Simple abdominal pain may be the sole symptom.

Causes:

- Alcohol

- Gallstones

- Metabolic disorders: hereditary pancreatitis, hypercalcemia, elevated triglycerides, malnutrition

- Abdominal trauma

- Penetrating ulcers

- Carcinoma of the head of pancreas, and other cancer

- Drugs: diuretics (e.g., thiazides, furosemide)

- RadiationX-ray

- Autoimmune pancreatitis.

- Heart valve surgery ${ }^{[3]}$

- Fat necrosis

- Pregnancy

- Infections other than mumps,including varicella zoster

- Repeated marathon running

- Hyperparathyroidism

- Valproic acid

- Cystic fibrosis ${ }^{[4]}$

\section{Risk Factors for Pancreatitis:}

Pancreatitis can happen to anyone, but it is more common in people with certain risk factors. ${ }^{[1]}$

Risk factors of acute pancreatitis include:

- Gallstones

- Heavy alcohol drinking

- Acute pancreatitis may be the first sign of gallstones. Gallstones can block the pancreatic duct, which can cause acute pancreatitis. ${ }^{[1]}$

\section{Pancreatitis laboratory Diagnosis:}

- Elevated serum amylase and lipase levels, in combination with severe abdominal pain, often trigger the initial diagnosis of acute pancreatitis. However, they have no role in assessing disease severity.

- Serum lipase rises 4 to 8 hours from the onset of symptoms and normalizes within 7 to 14 days after treatment.[5]

\section{Treatment for acute pancreatitis:}

People with acute pancreatitis are typically treated with IV fluids and pain medications in the hospital. In some patients, the pancreatitis can be severe and they may need to be admitted to an intensive care unit (ICU). In the ICU, the patient is closely watched because pancreatitis can damage the heart, lungs, or kidneys. Some cases of severe pancreatitis can result in death of pancreatic tissue. In these cases, surgery may be necessary to remove the dead or damaged tissue if an infection develops. ${ }^{[1]}$

PT test measures the clotting time of plasma in the presence of an optimal concentration of tissue extract (thromboplastin) and indicate the overall efficiency of the extrinsic clotting system. Although originally thought to measure prothrombin, the test is now known to depend also on reaction with factors V,VII and X on the fibrinogen concentration of the plasma. ${ }^{[6]}$ PTT test measures the clotting time of plasma after the activation of contact Factors but without added tissue thromboplastin , and so indicates the overall efficiency of intrinsic pathway .To standardize the activation of contact factors ,the plasma is first pre-incubated with kaolin .A standardized phospholipid is provided to allow the test to be performed on platelet-poor plasma .The test depends not only on the contact factors and on factors VIII and IX , but also on the reactions with factors X,V, Prothrombin and fibrinogen. It is also sensitive to presence of circulating anticoagulants (inhibitors) and heparin. ${ }^{[7]}$ A platelet count may be requested to investigate abnormal skin and mucosal bleeding which can occur when the platelet count is very low (usually below $20 \times 10^{9} / 1$ ). Platelet counts are also performed when patients are being treated with cytotoxic drugs or other drugs which may cause thrombocytopenia. ${ }^{[8]}$ 


\section{Materials And Methods}

This study is a case control study, conducted in Khartoum, Sudan, in the period from March to April 2017 . Sixty samples were included in this study to assess the Prothrombin Time, Activated Partial Thromboplastic Time and Platelets count in acute pancreatitis Sudanese patients. Thirty samples were collected from patients (case group) and 30 samples were collected from healthy persons which represent control group. Five $\mathrm{ml}$ of blood were collected from each subject by clean venous puncture, $3 \mathrm{ml}$ of which was placed in an EDTA container for assessment of platelet count and other two $\mathrm{ml}$ was placed in Sodium citrate anticoagulant for measurement of PT and PTT. This study was approved by ethical committee of ministry of health, and informed consent was obtained from each participant before sample collection.Data was be collected by a structured questionnaire system from patients in IbnSina hospital. Patients' data were analyzed by SPSS computer program version 21 .

\section{PT AND PTT ASSESSMENT:}

PT was measured by delivering $0.1 \mathrm{ml}$ of patient platelet poor plasma in to containing stir in semiautomated coagulometer (COATRON-MI) diagnostic stage, $0.2 \mathrm{ml}$ was added of classified thromboplastin(CAT NO:PT200150) by automatic pipette, then pressing start at that moment. The machine recorded the measurement time at the moment which the clot was formed.APTT was measured by delivering 0.1 $\mathrm{ml}$ of patient platelet poor plasma in to containing stir in semi-automated coagulometer (COATRON-MI) diagnostic stage, $0.1 \mathrm{ml}$ of the Kaolin-phospholipids solution (CAT NO: APTT202180) was added by automatic pipette and start the stopwatch simultaneously after 3 minutes added $0.1 \mathrm{ml}$ of CaCL2 then pressing start at that moment. The machine recorded the measurement time at the moment which the clot was formed.

\section{PLATELETS COUNT ASSESSMENT:}

Was measured using Sysmex Kx-21N

AMYLASE LEVEL ASSESSMENT:

The amylase level was taken from the patients records the will be measured using Biosystem Chemistry Analyzer .

\section{Results}

The results of this study showed thatin total of 60 samples included in this study, the males were $23(76.6 \%)$ and females were 7 (23.4\%) in patients group. (Figure1)

The results showeds:-

The mean \pm SD and P.value of age, amylase ,PT, PTT and Platelets in case group were $59.33( \pm 7.36), 226.79 \pm(14.97), 31.06( \pm 6.29)$ seconds, $57.33( \pm 9.20)$ seconds and $306.53( \pm 60.44) \times 10^{9} / \mu \mathrm{L}$ with P.value $(\mathrm{P}=0.479)$ respectively, While the mean \pm SD of PT, PTT and Platelets in control group were 13.33 $( \pm 1.15)$ seconds, $34.94( \pm 2.59)$ seconds and $287.43( \pm 47.59) \times 10^{9} / \mu \mathrm{L}$ respectively with P.value $(\mathrm{P}=0.00)$. (Table 1).

The association between genders and mean \pm SDof PT,PTT and platelets in case group were $30.65( \pm 6.32)$ seconds, $57.43( \pm 9.75)$ secondsand $300.60( \pm 63.02) \times 10^{9} / \mu \mathrm{L}$ respectively in males and $32.42( \pm 6.47)$ seconds, $57.00( \pm 7.81)$ seconds and $326.00( \pm 50.20) \times 10^{9} / \mu \mathrm{L}$ respectively in females.(Table 2$)$.

The correlation between age and PT, PTT and platelets in case group were $(0.351),(0.496),(-0.067)$ respectively.(Table 3).

The correlation between amylase and PT, PTT and platelets in case group were $(0.031),(0.104),(0.082)$ respectively.(Table 4).

Figure 1: Shows gender distribution in case group

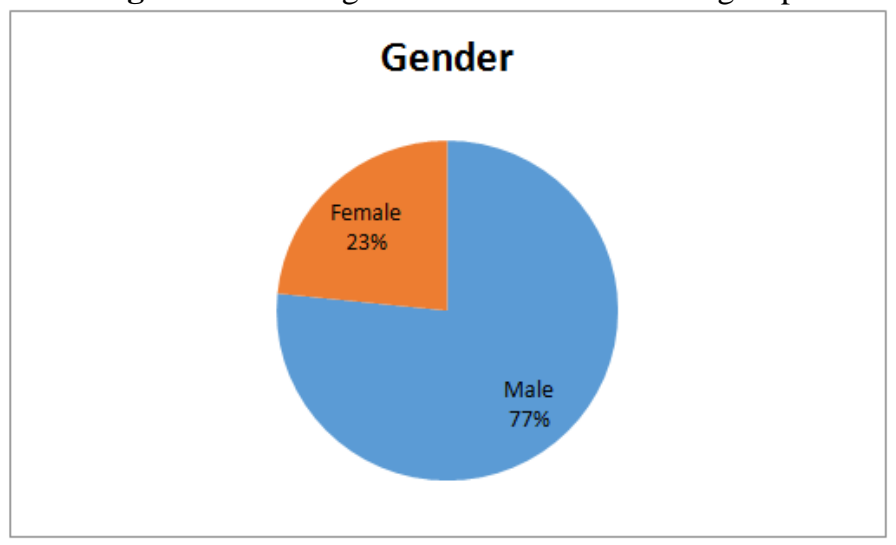


Assessment of Prothrombin Time, Activated Partial Thromboplastic Time Platelets count and ..

Table 1: Shows the means of PT, PTT and platelets counts among case and control groups $(\mathrm{P}=0.00)$

\begin{tabular}{|l|l|l|l|}
\hline & Case $( \pm$ SD $)$ & Control $( \pm$ SD) & P. value \\
\hline PT & $31.06( \pm 6.29)$ & $13.33( \pm 1.15)$ & 0.000 \\
\hline Aptt & $57.33( \pm 9.20)$ & $34.94( \pm 2.59)$ & 0.000 \\
\hline Platelets & $306.53( \pm 60.44)$ & $287.43( \pm 47.59)$ & 0.000 \\
\hline
\end{tabular}

Table 2: Shows the association between genders and PT, PTT and platelets in case group.. (P>0.05)

\begin{tabular}{|l|l|l|l|}
\hline & Male $( \pm$ SD) & Female $( \pm$ SD) & P. value \\
\hline PT $($ Sec.) & $30.65( \pm 6.32)$ & $32.42( \pm 6.47)$ & 0.523 \\
\hline PTT $($ Sec. $)$ & $57.43( \pm 9.75)$ & $57.00( \pm 7.81)$ & 0.915 \\
\hline Platelets $(\mathbf{x ~ 1 0} / \boldsymbol{\mu L})$ & $300.60( \pm 63.02)$ & $326.00( \pm 50.20)$ & 0.339 \\
\hline
\end{tabular}

Table 3: Shows the correlation between age and PT, PTT and platelets in case group.. $(\mathrm{P}>0.05)$

\begin{tabular}{|l|l|l|}
\hline & $\mathrm{R}$ & P.value \\
\hline PT & 0.351 & 0.057 \\
\hline PTT & 0.496 & 0.05 \\
\hline Platelets & -0.067 & 0.725 \\
\hline **Correlation is significant at the 0.05 level (2-tailed). & \multicolumn{2}{|l}{} \\
\hline
\end{tabular}

Table 4: Shows the correlation between amylase and PT, PTT and platelets in case group. $(\mathrm{P}>0.05)$

\begin{tabular}{|l|l|l|}
\hline Amylase & $\mathrm{R}$ & $\mathrm{P}$. value \\
\hline PT & 0.031 & 0.871 \\
\hline aPTT & 0.104 & 0.581 \\
\hline Platelets & 0.082 & 0.668 \\
\hline$* *$ Correlation is significant at the 0.05 level (2-tailed). & \\
\hline
\end{tabular}

\section{Discussion}

Our results showed that the mean PT, PTT and Platelets among Sudanese patients with acute pancreatitis compared to normal subjects (control group) were significantly increased $(\mathrm{P}=0.000)$. These findings may be due to circulating factors originating fromthe inflamed pancreas that may damage various organsby variable mechanisms. The pancreatic disorders are responsible for significant alteration in coagulation system.

Those findings are incorcondance with previous study the findings conducted by Sana Eltahir et al ${ }^{[9]}$ which found raised in PT 14.9( \pm 3.8$)$ seconds , PTT 40.5( \pm 10.7$)$ seconds and normal platelets count 244.9 $( \pm 124.4) \times 10^{9} / \mu \mathrm{L}$. These findings also incorcondance with Saif NW. ${ }^{[10]}$ The results of his study revealed abnormal prothrombin time and partial thromboplastin time.

The role of platelets in pathophysiology of disease hasnot been elucidated yet. There is activation of plateletsduring acute pancreatitis and alteration of plateletnumbers and indexes between onset and remission ofthe disease which reflect the bone marrow response. ${ }^{[11]}$ Platelets are directly involved in the systemic inflammatory process of acute pancreatitiswhich leads to consumption of platelets and arecompensated by an immediate bone marrow response. This study also showed therewere insignificant differences between gender and coagulation parameters (PT, PTT and platelets) $(\mathrm{P}>0.05)$. And showed insignificant correlation between the amylase and coagulation parameters (PT, PTT and platelets) $(\mathrm{P}>0.05)$.

\section{Conclusion}

This study concluded that PT and APTT were prolonged in patients with acute pancreatitis and normal platelets count.

\section{Recommendations}

Further studies containing large numbers of samples.

\section{Acknowledgement}

I dedicate this work to my mother, my father, my brothers and sisters, my friends and supervisor.

\section{References}

[1]. Melinda Ratini, DO, MS, WebMD Medical Reference on September 25, 2015

[2]. Chung, Joo Won; Ryu, Sung Ho; Jo, Jung Hyun; Park, JeongYoup; Lee, Sak; Park, Seung Woo; Song, Si Young; Chung, Jae Bock "Clinical Implications and Risk Factors of Acute Pancreatitis after Cardiac Valve Surgery". Yonsei Medical Journal. (2013). 54 (1): 154.

[3]. Gumaste V, Dave P, Weissman D, Messer J (1991). "Lipase/amylase ratio. A new index that distinguishes acute episodes of alcoholic from nonalcoholic acute pancreatitis". Gastroenterolog, (1991),101 (5): 1361-6.

[4]. A.V. Hoffbrand, J. E. Pettit, P. A. H. Moss. Essential haematology, 5th edition. USA, Blackwell publishing Ltd, $2006: 264-302$.

[5]. Sir John V .Dacie/S.M.Lewis ,Practical Haematology, Eighth Edition, New York 1995.

[6]. Monica cheesbrough, Distric laboratory practice in tropical countries, part $2.2^{\text {nd }}$ edition, Newyork,2009.

[7]. Worthing-biochem.com, Effects of PH (Introduction to Enzymes) Retrieved 17 May 2015. 
Assessment of Prothrombin Time, Activated Partial Thromboplastic Time Platelets count and ..

[8]. Sana Eltahir Abdalla1, Hana Tajelsir Adam2 and EnaamAbdelrahmanAbdelgadir ,Coagulation Profile (PT, APTT, fibrinogen level and platelets count) in Sudanese patients with acute pancreatitis. Merit Research Journal of Microbiology and Biological Sciences Vol,(2013), 2(1) pp. 001-004.

[9]. -Murphy D, Imrie CW, Davidson JF (1977). Haematological abnormalities in acute pancreatitis. A prospective study. Postgrad Med J. Jun; (1977) 53(620):310-4.

[10]. -Badhal SS1, Sharma S, Saraya A, Mukhopadhyay AK (2012). Prognostic significance of D-dimer, natural anticoagulants and routine coagulation parameters in acute pancreatitis. Trop Gastroenterol. (2012), 33(3):193-9.

[11]. Akbal E1, Demirci S, Koçak E, Köklü S, Başar O, Tuna Y ,Alterations of platelet function and coagulation parameters during acute pancreatitis. Blood Coagul Fibrinolysis, (2013), 24(3):243-6. 